\title{
Performance Evaluation of ANFIS Network in Determination of Inverse Kinematic Equations for a 4DOF Robotic Arm
}

This paper was downloaded from TechRxiv (https://www.techrxiv.org).

\section{LICENSE}

CC BY-NC-SA 4.0

SUBMISSION DATE / POSTED DATE

$12-02-2022$ / 22-02-2022

CITATION

Udosen, David (2022): Performance Evaluation of ANFIS Network in Determination of Inverse Kinematic Equations for a 4DOF Robotic Arm. TechRxiv. Preprint. https://doi.org/10.36227/techrxiv.19165388.v1

$\mathrm{DOI}$

10.36227/techrxiv.19165388.v1 


\title{
Performance Evaluation of ANFIS Network in Determination of Inverse Kinematic Equations for a 4DOF Robotic Arm
}

\author{
David Udosen \\ Electrical and Electronics Engineering Department, School of Engineering and Engineering Technology, Federal \\ University of Technology Akure, Nigeria \\ davidudosen@gmail.com
}

\begin{abstract}
The paper evaluates the ability of the Adaptive Neuro-Fuzzy Inference System (ANFIS) Network to develop its own set of inverse kinematics (IK) equations when trained with forward kinematics data. The forward kinematics equation is developed using the Denavit-Hartenberg (D-H) approach while the inverse kinematics equations (which is used to test the ANFIS Network) is developed analytically using geometry. This evaluation is carried out on a four degree of freedom (4DOF) robotic arm.
\end{abstract}

Keywords - Degree of freedom (DOF), Neuro-Fuzzy Systems, ANFIS

\section{Introduction}

The study of the kinematics of robotic manipulators is one of the most vital areas in the field of robotics. Establishing a mechanism that determines the relationship between manipulator joint angles and Cartesian coordinates of the position and orientation of the manipulator's tool-point, is the central theme of robotic kinematics. Based on initial work by Denavit and Hartenberg [1], a standard process of finding solutions to forward kinematics problems has been developed. Presently, the process of allocating different parameters to every robot joint as well as obtaining the position and orientation of end-effector with respect to a base frame has been perfected and is well documented.

In the industry, the trajectory planning for tasks such as precision assembly, packaging and spot welding is done in the background (termed offline mode) in order to determine the Cartesian path for successful task execution. Hence, conversion of Cartesian coordinates into joint angles using IK relations is necessary for the determination of the robot's Cartesian path. This shows the level of importance of IK computation in the design, path-planning workspace analysis, trajectory planning and control of robots.
Review of similar papers has shown that various methods of obtaining solution of IK problems has been attempted. Methods such as numerical methods [2] [3] [4] [5], geometric methods [6] [7], algebraic methods [8] [9] or a combination of two methods [10] have been used in the control of a robot manipulator. Generally, when closed form solutions are desired, algebraic methods are used but still, they do not guarantee closed form solutions. Closed form solutions must exist geometrically for the first three joints of a robot manipulator when using geometric methods. Initial value selection, absence of multiple solutions, and limited convergence are factors that must be present before numerical methods are used. Furthermore, if a particular pose of a robot yields a singular Jacobian matrix, numerical methods cannot be used. Above all, popularly used industrial robots that have coupled position and orientation kinematics prove problematic when trying to develop accurate IK solutions for them using both algebraic and numerical methods. The reason being that the trigonometric equations derived from manipulator analysis are often complex and as such closed form solutions are not obtainable.

In summary, it is evident that finding IK solutions of robot manipulator effectively using the traditional geometric, numeric or algebraic approach pose difficulties. In addition, none of these methods present a standard procedure for obtaining IK solutions that is independent of the number of degrees of freedom and the robot's geometry. The quest for a robust method of obtaining IK solutions that incorporates a standard procedure is ongoing. Methods involving fuzzy logic and artificial neural network have been attempted.

The learning ability of neural networks was combined with the very-high-level-language information processing capability of Fuzzy Inference Systems (FIS) to yield systems that could easily be manipulated by end users and had great modelling abilities. These systems were named Neuro-Fuzzy systems. 
In this paper, the ANFIS [11] performance is evaluated in solving the IK problem of a $4 \mathrm{DOF}$ robot arm. The paper is organized as follows: the ANFIS architecture is presented in section II. Section III borders on evaluation and results and section IV contains the conclusion.

\section{Architecture of ANFIS}

\section{A. ANFIS Structure}

Through a learning algorithm that optimizes the parameters of a given FIS, the ANFIS performs the mapping relation between the input and output data. The ANFIS architecture has a five-layered structure. This includes: a fuzzy layer, product layer, normalized layer, de-fuzzy layer and the summation layer. Figure 1 shows a typical ANFIS architecture. A fixed node is represented by a circle while a square represents an adjustable node. To illustrate this point, let $e$ and $f$ be two inputs with $g$ as the output in the FIS. The Mamdani [12] [13] and the Sugeno [14] fuzzy models are the only models supported by MATLAB. The ANFIS used in this paper implements a first-order Sugeno FIS. Some of the reasons that make the Sugeno fuzzy model a better option for development of IK solutions include: constant or linear outputs, high interpretability, computational efficiency, and built-in optimal and adaptive techniques.

The rule set for a first-order Sugeno fuzzy system can be expressed as:

Rule 1: if $e$ is $L_{l}$ and $f$ is $M_{l}$, then $g_{l}=a_{l} e+b_{l} f+c_{l}$

Rule 2: if $e$ is $L_{2}$ and $f$ is $M_{2}$, then $g_{2}=a_{2} e+b_{2} f+c_{2}$

where $L_{i}$ and $M_{i}$ are the fuzzy sets in the antecedent, $a_{i}$, $b_{i}$, and $c_{i}$ are parameters assigned during training. It can be seen from Figure 1 that all the ith nodes of the first layer are adaptive nodes with node output expressed as:

$$
\begin{aligned}
& O_{i}^{1}=\mu_{L_{i}}(e), i=1,2 \\
& O_{i}^{1}=\mu_{M_{i-2}}(f), i=3,4
\end{aligned}
$$

where $\mu_{L_{i}}(e)$ and $\mu_{M_{i-2}}(f)$ can adopt any fuzzy membership function (MF). However, this paper uses the Gaussian MF. The symmetric Gaussian function gaussmf depends on two parameters $\sigma$ and $c$ as given by: $\operatorname{gaussm} f(x ; \sigma, c)=e^{\frac{-(x-c)^{2}}{2 \sigma^{2}}}$

where $\left\{\sigma_{i}, c_{i}\right\}$ is the parameter set that modifies the shapes of the MF. The parameters of this layer are termed the premise parameters.
The nodes in the second layer are all fixed nodes and are labelled $\Pi$. The output of the nodes in this layer is a product of all incoming signals [15]:

$$
O_{i}^{2}=\omega_{i}=\mu_{L_{i}}(e) \mu_{M_{i}}(f), \quad i=1,2
$$

Each node output represents the firing strengths of a reasoning rule.

The third layer is made up of fixed nodes (labelled N). In this layer, each of the firing strengths of the reasoning rule are compared with the sum of all the firing strengths. These normalized firing strengths are computed in this layer as:

$$
O_{i}^{3}=\bar{\omega}_{i}=\frac{\omega_{i}}{\omega_{1}+\omega_{2}}, \quad i=1,2
$$

where $\bar{\omega}_{i}$ is referred to as the normalized firing strengths.

All $i$ th node in Layer 4 are adaptive nodes and have the following node function:

$$
O_{i}^{4}=\bar{\omega}_{i} g_{i}=\bar{\omega}_{i}\left(a_{i} e+b_{i} f+c_{i}\right), \quad i=1,2
$$

where $\bar{\omega}_{i}$ is third layer's output with parameter set $\left\{a_{i}, b_{i}, c_{i}\right\}$. Parameters of Layer 4 are referred to as consequent parameters.

With label $\Sigma$, Layer 5 is a single fixed node that sums up all the incoming inputs to yield the overall output. This is given by the function below:

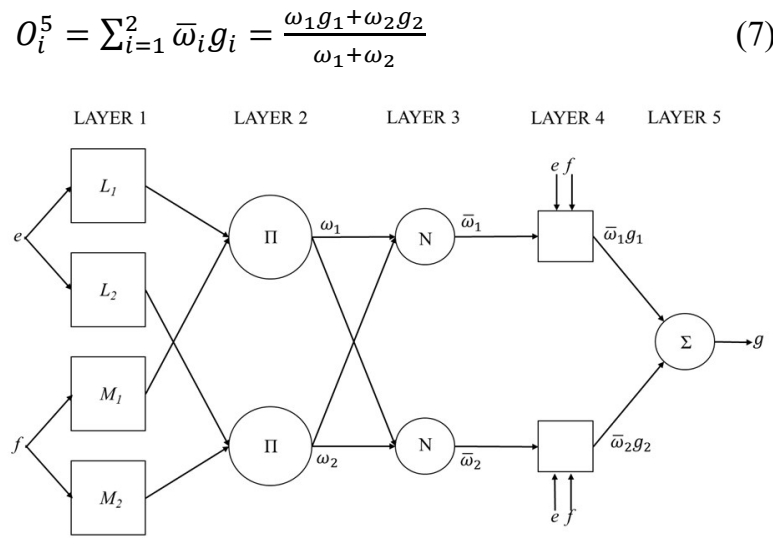

Figure 1: ANFIS Architecture 


\section{B. Learning Algorithms}

From the ANFIS architecture, it can be deduced that when the premise parameter values are fixed, the output of the ANFIS can be calculated as:

$$
g=\frac{\omega_{1}}{\omega_{1}+\omega_{2}} z_{1}+\frac{\omega_{2}}{\omega_{1}+\omega_{2}} z_{2}
$$

Substituting (5) into (8):

$$
g=\bar{\omega}_{1} g_{1}+\bar{\omega}_{2} g_{2}
$$

Substituting the if-then rules into (9), it becomes:

$$
g=\bar{\omega}_{1}\left(a_{1} e+b_{1} f+c_{1}\right)+\bar{\omega}_{2}\left(a_{2} e+b_{2} f+c_{2}\right)
$$

The output can be shown to be a linear combination of the consequent parameters. Rearranging (10):

$$
\begin{aligned}
g= & \left(\bar{\omega}_{1} e\right) a_{1}+\left(\bar{\omega}_{1} f\right) b_{1}+\left(\bar{\omega}_{1}\right) c_{1}+\left(\bar{\omega}_{2} e\right) a_{2}+ \\
& \left(\bar{\omega}_{2} f\right) b_{2}+\left(\bar{\omega}_{2}\right) c_{2}
\end{aligned}
$$

The Least Square Method (LSM) can be used to determine optimal values of the consequent parameters. With adaptive premise and consequent parameters, the search area becomes larger and the convergence of training is slower. To solve this problem, the LSM and the backpropagation algorithm is combined in an algorithm called the hybrid learning algorithm [16]. With this algorithm the dimension of the search area of the backpropagation algorithm is decreased hereby increasing its rate of convergence. The premise and consequent parameters are modified during the learning procedure until the desired FIS response is achieved.

There are two steps involved in the hybrid learning algorithm. These are the forward and the backward passes. For the forward pass, the premise parameters are fixed while the network inputs are transported forward till Layer 4 where the consequent parameters are recognized by the LSM. Conversely, for the backward passes, the consequent parameters are fixed and the error signals are transported from the output end to the input end with the backpropagation algorithm updating the premise parameters. The ANFIS training procedure is shown in Figure 2.

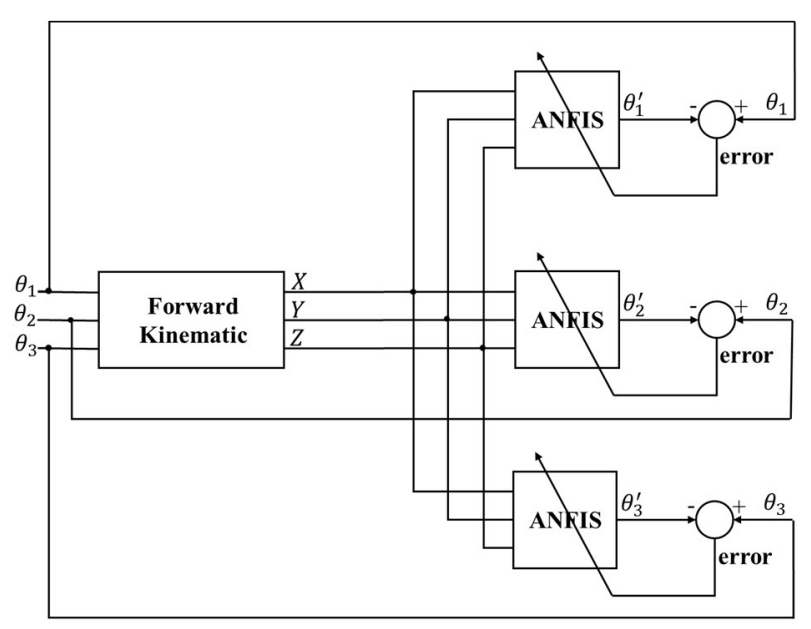

Figure 2: ANFIS training procedure

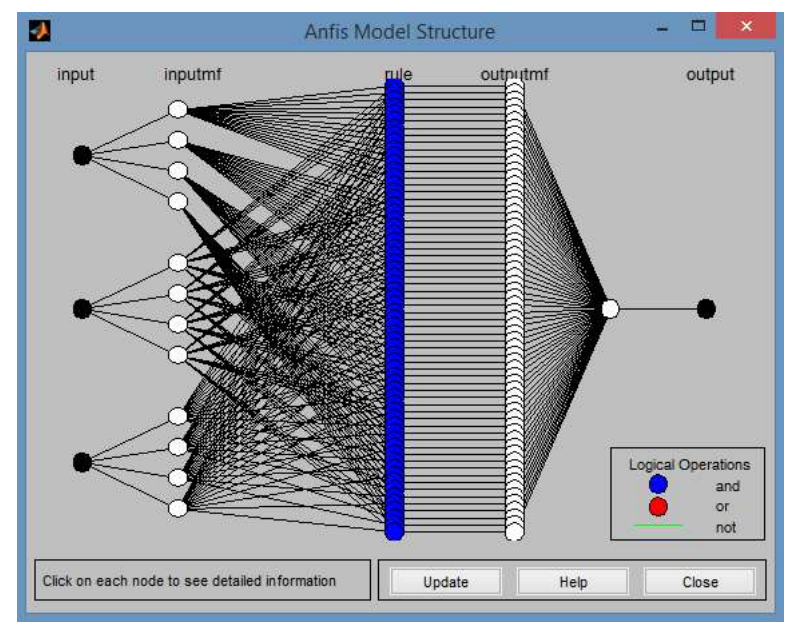

Figure 3: Model structure of an ANFIS Network with 4 Membership Functions

\section{Evaluation and Results}

\section{A. Kinematics of the Robotic Arm}

The performance of the trained ANFIS network will be verified in this section. A robotic arm purchased from Microscale Embedded was used for our simulation. The arm possesses four degrees of freedom. However, only three of these joints are involved in the positioning of the arm, all of which are revolute joints.

Figure 4 is a picture of the robotic arm with its corresponding Denavit-Hartenberg (D-H) parameters given in Table 1. Forward kinematics is used in this paper to obtain the data that is used to train the ANFIS Network. 


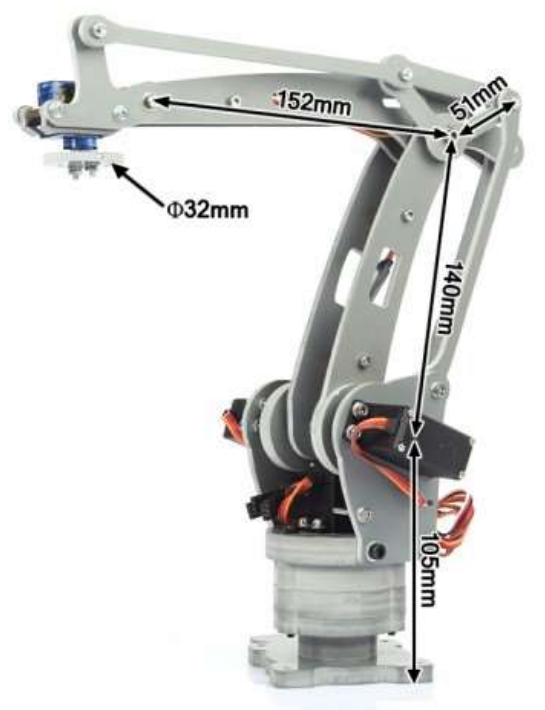

Figure 4: Microscale Embedded Robotic Arm

Table 1: Denavit-Hartenberg (D-H) Parameters of the Robotic Arm

\begin{tabular}{c|c|c|c|c|}
\hline & $\mathbf{a}_{\mathbf{i}-\mathbf{1}}$ & $\boldsymbol{\alpha}_{\mathbf{i}-\mathbf{1}}$ & $\mathbf{d}_{\mathbf{1}}$ & $\boldsymbol{\theta}_{\mathbf{1}}$ \\
\hline $\mathbf{1}$ & 0 & 0 & 0 & $\theta_{1}$ \\
\hline $\mathbf{2}$ & 0 & 90 & 0 & $\theta_{2}$ \\
\hline $\mathbf{3}$ & $1_{3}$ & 0 & 0 & $\theta_{3}$ \\
\hline $\mathbf{4}$ & 0 & -90 & $1_{4}$ & $\theta_{4}$ \\
\hline $\mathbf{5}$ & 0 & 0 & 0 & $\theta_{5}$ \\
\hline
\end{tabular}

The overall forward kinematic matrix for the robot arm is given in Equation (12).

$$
\begin{aligned}
& { }_{5}^{0} T={ }_{4}^{0} T_{5}^{4} T={ }_{3}^{0} T_{4}^{3} T_{5}^{4} T={ }_{2}^{0} T_{3}^{2} T_{4}^{3} T_{5}^{4} T={ }_{1}^{0} T_{2}^{1} T_{3}^{2} T_{4}^{3} T_{5}^{4} T \\
& =\left[\begin{array}{cccc}
a_{11} & a_{12} & a_{13} & l_{4}\left(-c_{12} s_{3}-c_{13} s_{2}\right)+l_{3} c_{12} \\
a_{21} & a_{22} & a_{23} & l_{4}\left(s_{1} c_{23}-s_{123}\right)+l_{3} s_{1} c_{2} \\
a_{31} & a_{32} & a_{33} & l_{3} s_{2} \\
0 & 0 & 0 & 1
\end{array}\right](12)
\end{aligned}
$$

where:

$$
\begin{aligned}
& l_{3}=140 \mathrm{~mm} \\
& l_{4}=152 \mathrm{~mm} \\
& a_{11}=c_{5}\left[c_{4}\left(c_{123}-c_{13} s_{2}\right)-s_{14}\right]-s_{5}\left[s _ { 4 } \left(c_{123}-\right.\right. \\
& \left.\left.c_{1} s_{23}\right)-s_{1} c_{4}\right] \\
& a_{12}=-s_{5}\left[c_{4}\left(c_{123}-c_{13} s_{2}\right)-s_{14}\right]-c_{5}\left[s _ { 4 } \left(c_{123}-\right.\right. \\
& \left.\left.c_{1} s_{23}\right)-s_{1} c_{4}\right]
\end{aligned}
$$

$$
\begin{aligned}
& a_{13}=-c_{12} s_{3}-c_{13} s_{2} \\
& a_{21}=c_{5}\left[c_{4}\left(s_{1} c_{23}-s_{123}\right)+c_{1} s_{4}\right]-s_{5}\left[s _ { 4 } \left(s_{1} c_{23}-\right.\right. \\
& \left.\left.s_{123}\right)+c_{14}\right] \\
& a_{22}=-s_{5}\left[c_{4}\left(s_{1} c_{23}-s_{123}\right)+c_{1} s_{4}\right]-c_{5}\left[s _ { 4 } \left(s_{1} c_{23}-\right.\right. \\
& \left.\left.s_{123}\right)+c_{14}\right] \\
& a_{23}=-s_{13} c_{2}-s_{12} c_{3} \\
& a_{31}=c_{45}\left(s_{2} c_{3}+c_{2} s_{3}\right)-s_{45}\left(s_{1} c_{23}-s_{123}\right) \\
& a_{32}=-c_{4} s_{5}\left(s_{2} c_{3}+c_{2} s_{3}\right)-s_{4} c_{5}\left(s_{1} c_{23}-s_{123}\right) \\
& a_{33}=s_{23}+c_{23}
\end{aligned}
$$

Note that $s_{1}, s_{12}$ and $s_{123}$ represents $\sin \left(\theta_{1}\right)$, $\sin \left(\theta_{1}\right) \sin \left(\theta_{2}\right)$ and $\sin \left(\theta_{1}\right) \sin \left(\theta_{2}\right) \sin \left(\theta_{3}\right)$ respectively. The same can be said for $\cos$ which is represented by $c$.

The D-H parameters and the forward kinematics matrix are tools that are used to uniquely define robotic manipulators.

\section{B. Training Data Generation}

The forward kinematics relations provides the data necessary for training the ANFIS network. The joint space can be viewed as a reverse image of the Cartesian space and vice versa. Similarly, the inverse kinematics can be viewed as a reverse image of the forward kinematics and vice versa. Thus forward kinematic relations is used to determine the Cartesian coordinates of the robotic arm's tool point. The first three cells of the last column in the forward kinematic matrix represents the $x, y$, and $z$ coordinates respectively of the robotic arm's tool point. This was used in training the ANFIS Network.

Once the training was complete, the three ANFIS networks had learned to approximate the angles (theta1, theta2, theta3) as a function of the coordinates (x, y, z). One advantage of using the fuzzy approach was that the ANFIS network would now approximate the angles for coordinates that are similar but not exactly the same as it was trained with. For example, the trained ANFIS networks are now capable of approximating the angles for coordinates that lie between two points that were included in the training dataset. This will allow a controller that uses ANFIS to move the arm smoothly in the input space. 


\section{Validating the ANFIS Network}

Having trained the Network, an important follow up step is to validate the Network to determine how well the ANFIS Network would perform if it was employed in a larger control system.

Since the problem deals with a four DOF robotic arm whose inverse kinematic relations can easily be derived using geometry, it is possible to test the ANFIS network's solution with that calculated using geometry.

THETA1D, THETA2D and THETA3D are the variables that hold the values of $\theta_{1}, \theta_{2}$ and $\theta_{3}$ deduced using the inverse kinematics relations while THETAIP, THETA2P and THETA $3 P$ are the variables that hold the values of $\theta_{1}, \theta_{2}$ and $\theta_{3}$ predicted by the ANFIS Network.

\section{Results}

Figures 5, 6 and 7 are plot that show the difference between $\theta$ values deduced during the inverse kinematics calculation and that predicted by the trained ANFIS network.

The error difference in each of the $\theta$ values is shown in Table 2

Table 2: Summary of Error Difference between ANFIS $\theta$ Values and Calculated $\theta$ Values

\begin{tabular}{|l|c|c|c|}
\hline & $\theta_{1}$ & $\theta_{2}$ & $\theta_{3}$ \\
\hline Error & 5 & 0.2 & 2 \\
\hline
\end{tabular}

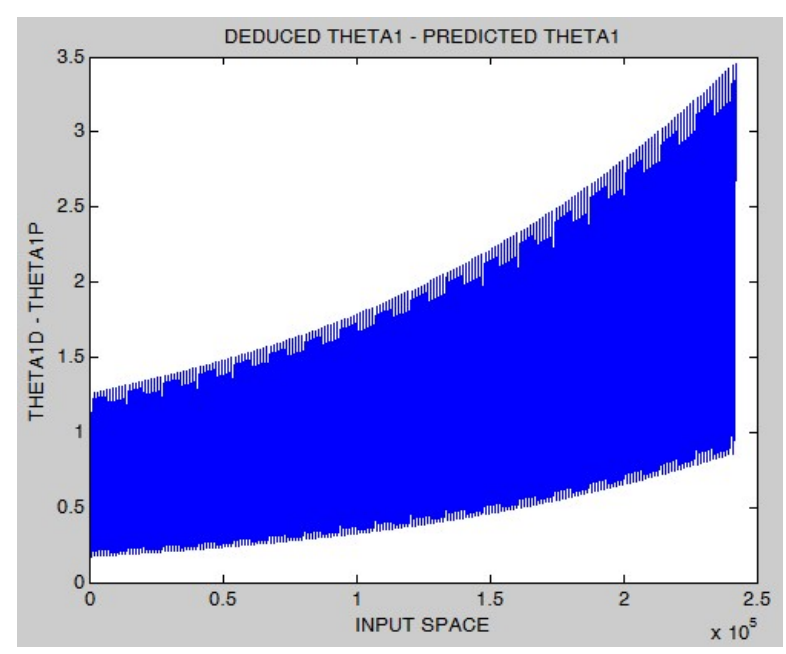

Figure 5: Error difference between ANFIS Values of $\theta_{1}$ and Calculated Values of $\theta_{1}$

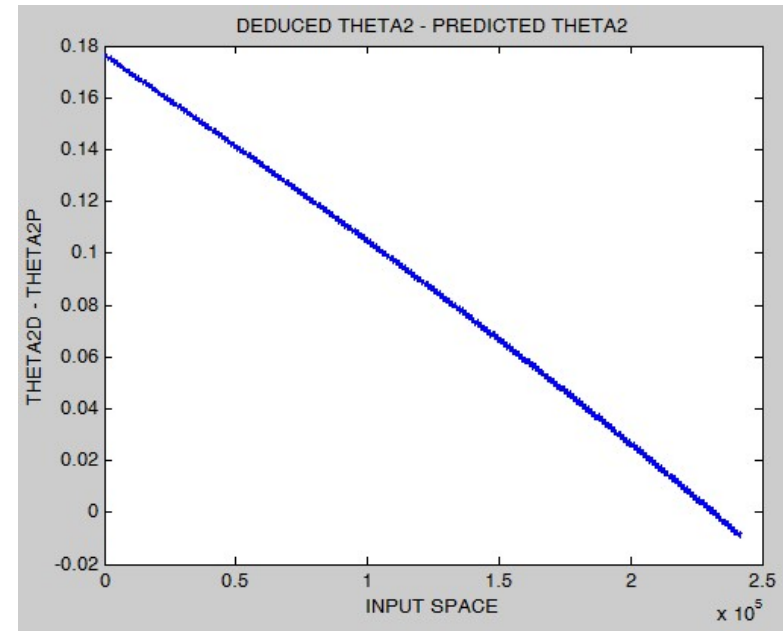

Figure 6: Error difference between ANFIS Values of $\theta_{2}$ and Calculated Values of $\theta_{2}$

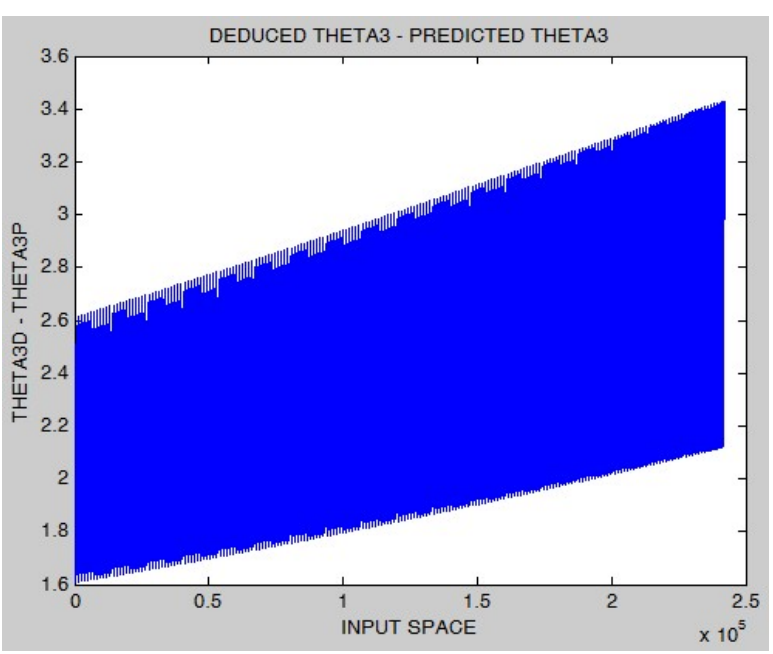

Figure 7: Error difference between ANFIS Values of $\theta_{3}$ and Calculated Values of $\theta_{3}$

\section{Conclusion}

The performance of an inbuilt MATLAB Neuro-fuzzy algorithm, ANFIS was tested. This was done by training the system with the forward kinematics equations of the robotic arm and allowing the ANFIS network to determine the inverse kinematics equations on its own. The derived inverse kinematics equations gotten from the system was then tested against the inverse kinematics equations that were calculated using geometry. The difference between these two were plotted on graphs. From the graphs it could be seen that the ANFIS Network performed well in determining the inverse kinematics equations and thus, can be used in control systems where $\theta$ values of very high precision are not important. 


\section{References}

[1] J. Denavit and R. Hartenberg, "A kinematic notation for lower-pair mechanisms based on matrices," ASME Journal of Applied Mechanics, pp. 215-221, 1995.

[2] L.-C. T. Wang and C. C. Chen, "A Combined Optimization Method for Solving the Inverse Kinematics Problem of Mechanical Manipulators," IEEE TRANSACTIONS ON ROBOTICS AND AUTOMATION, vol. 7, no. 4, pp. 489-499, 1991.

[3] C. W. I. Wampler, "Manipulator Inverse Kinematic Solutions Based on Vector Formulations and Damped Least-Squares Methods," IEEE TRANSACTIONS ON SYSTEMS, MAN, AND CYBERNETICS, VOL. SMC-16, NO. 1, , Vols. SMC-16, no. 1, pp. 93-101, JANUARY/FEBRUARY 1986.

[4] A. D. Luca and B. Siciliano, "Closed-Form Dynamic Model of Planar Multilink Lightweight Robots," IEEE TRANSACTIONS ON SYSTEMS, $M A N$, AND CYBERNETICS, vol. 21, no. 4, pp. 826-839, JULY/AUGUST 1991 .

[5] T. Kishimoto and Y. Fujimoto, "Numerically Stable Inverse Kinematics Calculation of Robot Manipulators even in Singular Configuration," in The 30th Annual Conference of the IEEE Industrial Electronlcs Soclety, Busan, Korea, November 2004.

[6] A. M. Sonda, M. B. R. Neila and D. Tarak, "Geometric Modeling of the Parallel Robot Par4 Using the Modified Denavit Hartenberg Method," in 17th international conference on Sciences and Techniques of Automatic control \& computer engineering, Sousse, Tunisia, December 2016.

[7] O. M. Omisore, S. R. L. Z. N. Han and L. Wang, "A Geometric Solution for Inverse Kinematics of Redundant Teleoperated Surgical Snake Robots," in Industrial Technology (ICIT), 2017 IEEE International Conference, Toronto, Canada, March 2017.

[8] V. A. d. S. Marques and R. S. Gonçalves, "Humanoid Robot's Inverse kinematics Using
Algebraic Geometry," in 2015 12th Latin American Robotics Symposium and 2015 Third Brazilian Symposium on Robotics, Uberlandia, Brazil, October 2015.

[9] S. Stifter, "Algebraic Methods for Computing Inverse Kinematics," Journal of Intelligent and Robotic Systems, vol. 11, pp. 78-89, 1994.

[10] C. W. Wampler and A. J. Sommese, "Numerical Algebraic Geometry and Algebraic Kinematics," January 2011.

[11] "ANFIS : Adaptive-Network-Based Fuzzy Inference System," IEEE TRANSACTIONS ON SYSTEMS, MAN, AND CYBERNETICS, vol. 23, no. 3, pp. 665-685, MAYIJUNE 1993.

[12] M. E. H., "Application of fuzzy algorithms for control of simple dynamic plant," PROCEEDINGS OF THE INSTITUTION OF ELECTRICAL ENGINEERS Control \& Science, vol. 121, no. 12, pp. 1585-1588, December 1974.

[13] C. Wang, "A Study of Membership Functions on MamdaniType Fuzzy Inference System for Industrial Decision-Making," Lehigh Preserve, Bethlehem, Pennsylvania, USA, 2015.

[14] M. SUGENO and T. TAKAGI, "MULTIDIMENSIONAL FUZZY REASONING," NorthHolland Publishing Company, Yokohama 227, Japan, May 1981.

[15] Z. M. O. Yousif and M. Fathelrahman, "NeuroFuzzy Inference System based Controller in 6 DoF in Puma 560," in Computing, Electrical and Electronics Engineering (ICCEEE), 2013 International Conference, Khartoum, Sudan, August 2013.

[16] A. Nedungadi, "Application of fuzzy logic to solve the robot inverse kinematic problem," Proceeding of 4th World Conference on Robotics Research, vol. 13, pp. 1-14, 1991.

[17] M. Aghajarian and K. Kiani, "Inverse Kinematics Solution of PUMA 560 Robot Arm Using ANFIS," The 8th International Conference on Ubiquitous Robots and Ambient Intelligence (URAI), pp. 574-578, 2011. 